\title{
Evaluation of Axial Length Changes after Combined Phacovitrectomy for Macula-off Rhegmatogenous Retinal Detachment
}

\author{
(1) Sadik Altan Ozal, ${ }^{1}$ (i) Ruveyde Garip, ${ }^{1}$ (i) Ece Ozal, ${ }^{2}$ (I) Ayca Kupeli ${ }^{1}$ \\ ${ }^{1}$ Department of Ophthalmology,Trakya University Faculty of Medicine, Edirne,Turkey \\ ${ }^{2}$ Department of Ophthalmology, Edirne State Hospital, Edirne,Turkey
}

\begin{abstract}
Objectives: To evaluate the reliability of optical biometry for correct intraocular lens (IOL) power calculation in eyes with macula-off rhegmatogenous retinal detachment (RRD) via comparing the axial lengths $(A L)$ measured with optical biometry preoperatively and postoperatively.

Methods: This retrospective study was conducted with 25 eyes of 25 patients who underwent combined surgery (phacoemulsification and pars plana vitrectomy (PPV)) with the diagnosis of RRD with macular involvement. Best-corrected visual acuity (BCVA), biomicroscopic examination, intraocular pressure measurement and full fundoscopic examination were performed in all patients. Axial length was measured using optical biometry in all patients. The AL measurements were repeated in the postoperative third month in the patients using gas tamponade during vitreoretinal surgery and in the third month after the silicone oil removal in patients using silicone-oil tamponade during vitreoretinal surgery.

Results: The mean age of the patients was $61.60 \pm 7.33$. Fifteen patients $(60 \%)$ were female, and $10(40 \%)$ were male. Postoperative BCVA values $(0.40 \pm 0.28)$ were significantly higher than the preoperative BCVA values $(0.14 \pm 0.22)(p<0.05)$. The preoperative mean value of $A L$ was measured as $24.66 \pm 1.90$ and the postoperative mean value of $A L$ was $24.67 \pm 1.91$. There was no statistically significant difference between the measured $A L$ values in the preoperative and postoperative periods $(p=0.258)$.

Conclusion: Combined surgery (phacoemulsification and PPV) is a frequently applied method in RRD treatment. The correct $A L$ measurement is important for the removal of refractive errors due to $A L$ measurement in patients with macula-off RRD. Optical biometry provides accurate results in these cases.
\end{abstract}

Keywords: Axial length, cataract, optical biometry, pars plana vitrectomy, retinal detachment.

\section{Introduction}

Rhegmatogenous retinal detachment (RRD) is the separation of the neurosensorial retina from the retinal pigment epithelium (RPE) through the retinal tear area of the liquefying vitreous (I). Pneumatic retinopexy, scleral buckling, or pars plana vitrectomy (PPV) can be used to treat RRD $(2,3)$. Currently, $P P V$ is preferred with the new developments in surgical instruments (4). A frequent complication of PPV surgery is cataracts with patients commonly needing cataract surgery for visual rehabilitation after PPV. Concomitant cataracts are also present in patients with RRD (5). Combined surgery (phacoemulsification and PPV) has become a common method in many vitreoretinal diseases as it eliminates the need for additional surgery and allows faster visual rehabilitation with a single healing process. It also provides better vitreous cleaning without concern for intraoperative lenticular contact or postoperative cataract progression (6-8).

Address for correspondence: Sadik Altan Ozal, MD. Trakya Universitesi Tip Fakultesi, Oftalmoloji Anabilim Dali, 22030, Edirne, Turkey

Phone: +90 5054504267 E-mail: altanozal@hotmail.com

Submitted Date: July 09, 2019 Accepted Date: November 26, 2019 Available Online Date: December 24, 2019

${ }^{\circ}$ Copyright 2019 by Beyoglu Eye Training and Research Hospital - Available online at www.beyoglueye.com OPEN ACCESS This work is licensed under a Creative Commons Attribution-NonCommercial 4.0 International License. 
Accurate calculation of the dioptry of the intraocular lens (IOL) used in cataract surgery is important for the postoperative visual outcome. Accurate measurement of axial length (AL) is more difficult in cases with macular detachment (7). In recent years, optical biometry has become widely used in AL measurements. Optical biometry works on the principle of partial coherent interferometry and provides more accurate results than ultrasonic AL measurements (9). While A-mode ultrasonography measures the distance between the corneal vertex and the vitreoretinal interface, optical biometry measures the distance between the corneal epithelium and RPE (10-12). Thus, in theory, if RPE is preserved, changes in the macula should not affect the AL measurements with optical biometry.

The present study aims to evaluate the reliability of optical biometry for accurate IOL power calculation and $A L$ measurement in patients with macula-off RRD.

\section{Methods}

Patients who underwent a PPV operation for macular involvement with RRD between January 2018 and January 2019 in the retina department of our clinic were included in this study. The data of all patients who underwent surgical treatment were retrospectively reviewed. Patients with corneal opacification that cannot be measured with optical biometry, any previous eye operation, age-related macular degeneration, diabetic retinopathy or glaucoma were excluded from this study. In addition, patients requiring more than one surgical procedure, recurrent retinal detachment and severe proliferative vitreoretinopathy were excluded. Patients without follow up after surgery were also excluded from this study. Informed patient consent was obtained preoperatively. The procedures of this study were approved by the institutional review board of the hospital and adhered to the tenets of the Declaration of Helsinki. The study protocol was approved by the local ethics committee (approval code: TUTF-BAEK 2019/28).

Detailed ophthalmologic examinations of the patients were performed preoperatively and postoperatively by the same experienced ophthalmologist (AK). Best-corrected visual acuity (BCVA) was measured with the Snellen chart. Biomicroscopic slit-lamp examinations were performed. Intraocular pressure (IOP) was measured with a Goldmann applanation tonometer (AT 900, Haag-Streit Diagnostics, Koeniz, Switzerland). A detailed fundus examination was conducted using a quadraspheric contact lens (Volk Optical Inc., Ohio, USA). Preoperative and postoperative optical coherence tomography (OCT) (Cirrus HD 5000, Carl Zeiss Meditec AG, Jena, Germany) were performed. All patients underwent preoperative $A L$ measurement with an
OCT-based optical biometry device (IOL Master 700, Carl Zeiss Meditec AG, Jena, Germany). AL measurements were repeated at the postoperative third month in patients using gas tamponade during vitreoretinal surgery and in the third month after the silicone oil removal in patients using silicone-oil tamponade during vitreoretinal surgery. The fundoscopic examination and OCT demonstrated that the retina and macula are attached in the postoperative period.

\section{Surgical Procedure}

All PPV operations were performed by the same surgeon (A.O.) under sterile conditions with general anaesthesia. All patients underwent combined phacovitrectomy (phacoemulsification and PPV) surgery. The operations were performed using an Infinity Constellation (Alcon, TX, USA) device. After intraocular lens (IOL) implantation into the bag, the main corneal incision was sutured with 10.0 nylon suture and three 23-gauge trocars were inserted from the pars plana level at $4 \mathrm{~mm}$ behind the limbus. Core vitrectomy and posterior hyaloid membrane peeling were applied. In all cases, a 25 -gauge trocar was inserted and a chandelier light source was placed. A 360-degree vitreous base cleaning was performed with a scleral depressor. The subretinal fluid was aspirated and 360-degree laser photocoagulation was applied to the peripheral retina. Gas (16\% octafluoropropane) or silicone oil (5000 cst) tamponade was used, and all sclerotomies were sutured with 8.0 polyglactin sutures. In the postoperative period, topical moxifloxacin and topical prednisolone acetate were given.

\section{Statistical Analysis}

The SPSS (Statistical Package for Social Sciences) program version 20.0 for Windows was used for statistical analysis in this study. Before performing calculations on the non-qualitative data, the Shapiro-Wilk test was used to determine the conformity of the data with a normal distribution. The Wilcoxon test was used to compare the numerical data when normal distribution could not be achieved. A p-value of less than 0.05 was accepted as statistically significant.

\section{Results}

In this retrospective study, 25 eyes of 25 patients who underwent PPV for macular involvement were included. Table I shows the baseline patient demographics. There were no complications related to cataract surgery.

The mean BCVA value was $0.14 \pm 0.22$ in the preoperative period. The mean BCVA measured in the postoperative third month in patients with gas tamponade, and in the third month after silicone oil removal in patients with silicone oil tamponade was $0.40 \pm 0.28$. Postoperative BCVA values were significantly higher than the preoperative BCVA values $(p<0.05)$. Table 2 shows the comparison of preoperative and 
Table I. Baseline patient demographics and characteristics

\begin{tabular}{lc} 
Parameter & Value \\
\hline Age & \\
Mean \pm SD & $61.6 \pm 7.33$ \\
Range & $41-82$ \\
Sex, $\mathrm{n}(\%)$ & \\
$\quad$ Men & $10(40.0)$ \\
Women & $15(60.0)$ \\
Tamponade type, $\mathrm{n}(\%)$ & \\
$\quad$ Silicone oil & $13(52.0)$ \\
Gas & $12(48.0)$
\end{tabular}

postoperative $\mathrm{AL}$ measurements.

The median preoperative spherical equivalent value was +0.37 ( $\min -8.25$ to $\max +4.50$ ). The median spherical equivalent value measured in the postoperative third month in patients with gas tamponade, and in the third month after silicone oil removal in patients with silicone oil tamponade was 0.00 ( $\min -0.50$ to $\max +1.75$ ). When the postoperative spherical equivalent values were examined, $24 \%$ of patients measured 0.00 dioptres, $16 \%-0.25$ dioptres, $16 \%+0.25$ dioptres, $12 \%-0.50$ dioptres, $4 \%+0.50$ dioptres, $4 \%+0.75$ dioptres, $12 \%+1.00$ dioptres, $4 \%+1.00$ dioptres, and $4 \%$ +1.75 dioptres (Fig. I).

Table 2. Comparison of the preoperative and postoperative axial length measurements

\begin{tabular}{lccc} 
& Preoperative & Postoperative & P \\
\hline Axial length & $24.66 \pm 1.90$ & $24.67 \pm 1.91$ & 0.258 \\
\hline
\end{tabular}

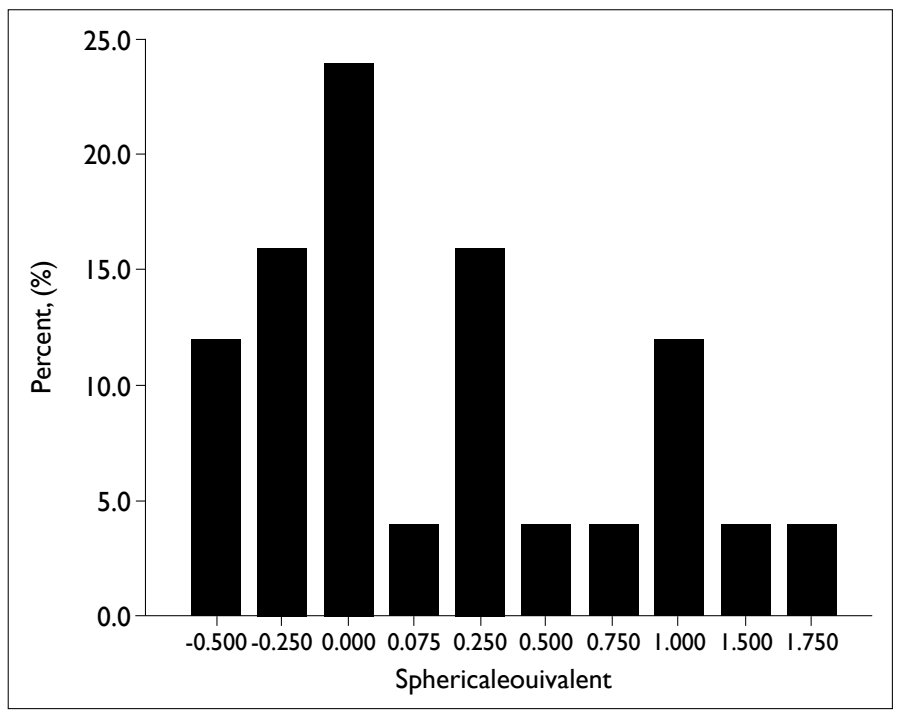

Figure I. Distribution of the postoperative spherical equivalent values.

\section{Discussion}

Pars plana vitrectomy is the most preferred surgical method in the treatment of RRD $(4,10)$. A frequent complication of PPV surgery is cataract with patients commonly requiring cataract surgery for visual rehabilitation after PPV. Concomitant cataracts are also present in patients with RRD (5). It has been reported that $75 \%$ of the patients develop a cataract within 10 years after PPV (I3). There is an increased risk of preoperative complications in cataract surgery in vitrectomised eyes. In these patients, anterior chamber fluctuations and deep anterior chamber increased mobility in the posterior lens, and zonular instability can be observed during cataract surgery due to the lack of vitreous support. During a vitrectomy, direct zonules or lens damage may develop, and the reactive effect of tamponade on zonules may cause zonular weakness (14). Any of these conditions may increase the risk of preoperative complications during cataract surgery. Therefore, combined surgery has become the preferred method. Phacovitrectomy has become common in many vitreoretinal diseases given that it allows for better visualisation of the vitreous by eliminating the need for additional surgery, provides faster visual rehabilitation with a single healing process, and does not lead to intraoperative lenticular contact or postoperative cataract progression (5-8).

Accurate calculation of the dioptric of the intraocular lens to be used in cataract surgery is very important concerning postoperative visual outcomes (7). Accurate measurement of $A L$ is a significant step in the accurate calculation of IOL power. AL measurement can be performed with ultrasonic biometry or the popular optical biometry devices. It has been reported that $50 \%$ of the postoperative refractive errors are due to axial length errors measured under ultrasound guidance (I5). Optical biometry works with the principle of partial coherence interferometry. Optical biometry has many advantages over ultrasonic biometry in $\mathrm{AL}$ measurements, such as a noncontact feature and fast, accurate, reproducible results $(15,16)$. While A-mode ultrasonography measures the distance between the corneal vertex and the vitreoretinal interface, optical biometry measures the distance between the corneal epithelium and RPE $(10,12)$. Thus, ultrasonic biometry measures $A L$ longer than optical biometry. Accurate measurement of the $A L$ is more difficult when calculating the IOL power in cases with macular detachment (7). Accurate AL measurements may not be obtained in these patients due to the difficulty of fixation and the distance between the cornea and the vitreoretinal interface $(8,9)$. Rahman et al. (5) evaluated the postoperative refractive status and reported that the lowest refractive errors were obtained using $A L$ measurements with optical biometry in RRD cases without macular involvement. The 
highest refractive errors were obtained with the use of $A L$ calculations measured by A-mode ultrasonography in RRD cases with macular involvement.

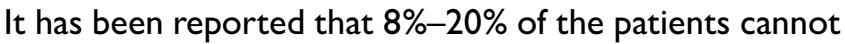
be measured with conventional optical biometry devices. Conventional biometry devices are unable to measure $A L$ in the presence of poor fixation, corneal opacity, advanced cataract, or media opacities (17, 18). The IOL Master 700 is a newly developed optical biometry device that can perform $\mathrm{AL}$ measurements in any of these conditions (19-20). In this study, AL measurements were performed with the IOL Master 700 device.

A one $\mathrm{mm}$ error in $\mathrm{AL}$ measurement leads to a three dioptre refractive change after cataract surgery (2I). In our study, no statistically significant difference was found between the $A L$ values measured by optic biometry preoperatively and after postoperative macular attachment. Additionally, postoperative refraction values were also consistent with preoperative target values. When we evaluated the postoperative spherical equivalent values, $72 \%$ of the patients were within the limits of emmetropia ranging from -0.50 to +0.50 . Only one patient had +1.75 dioptres postoperatively. The refractive values of the other patients ranged from +0.50 to +1.50 dioptres. These patients had high preoperative astigmatism. We thought our postoperative spherical equivalent values were high due to high residual astigmatism. We believe that all main corneal incisions are made from the superior quadrant and the suturing of the main corneal incisions induces postoperative astigmatism in phacoemulsification surgery. Our results are consistent with previous studies (5, 7-9). However, to our knowledge, there is no study showing the reliability of our device for the measurement of $A L$ in the macular involvement of retinal detachments in the current literature. Our results show that the Carl Zeiss IOL Master 700 device can be used safely for calculating the IOL power before phacovitrectomy surgery in patients with macula-off retinal detachments.

The limited number of cases and the absence of a control group are limiting factors in our study. In addition, the retrospective nature of this study limits our comparisons. A larger patient series is needed for clearer information.

In conclusion, combined phacovitrectomy is a common procedure in RRD surgery. The correct calculation of appropriate IOL power is critical for good visual rehabilitation after combined phacovitrectomy surgery. The accurate measurement of $A L$ is required for proper IOL power calculation. AL measured with the IOL Master 700 device is very accurate and can be used safely in combined surgeries.

\section{Disclosures}

Ethics Committee Approval: The research was approved by the ethics board of Trakya University (TÜTF-BAEK 2019/28).
Peer-review: Externally peer-reviewed.

Conflict of Interest: None declared.

Authorship Contributions: Involved in design and conduct of the study (SAO, RG, EO, AK); preparation and review of the study (SAO, RG, EO, AK); data collection (SAO, RG, EO, AK); and statistical analysis (SAO, RG, EO, AK).

\section{References}

I. Liao L, Zhu XH. Advances in the treatment of rhegmatogenous retinal detachment. Int J Ophthalmol 2019;12:660-7.

2. Shu I, Ishikawa H, Nishikawa H, Morikawa S, Okamoto F, Sakamoto T, et al. Scleral buckling versus vitrectomy for young Japanese patients with rhegmatogenous retinal detachment in the era of microincision surgery: real-world evidence from a multicentre study in Japan. Acta Ophthalmol 2019;97:e736e74I. [CrossRef]

3. Vidne-Hay O, Abumanhal M, Elkader AA, Fogel M, Moisseiev J, Moisseiev E. Outcomes of rhegmatogenous retinal detachment repair after failed pneumatic retinopexy. Retina 2019. [CrossRef]

4. Eibenberger K, Georgopoulos M, Rezar-Dreindl S, SchmidtErfurth U, Sacu S. Development of surgical management in primary rhegmatogenous retinal detachment treatment from 2009 to 2015. Curr Eye Res 2018;43:5 17-25. [CrossRef]

5. Rahman R, Bong CX, Stephenson J. Accuracy of intraocular lens power estimation in eyes having phacovitrectomy for rhegmatogenous retinal detachment. Retina 2014;34:14I5-20.

6. Sizmaz S, Esen E, Isik P, Cam B, Demircan N. Outcome and complications of combined phacoemulsification and 23-Gauge pars plana vitrectomy. J Ophthalmol 2019;2019:7918237. [CrossRef]

7. Rahman R, Kolb S, Bong CX, Stephenson J. Accuracy of user-adjusted axial length measurements with optical biometry in eyes having combined phacovitrectomy for macular-off rhegmatogenous retinal detachment. J Cataract Refract Surg 2016;42:1009-14. [CrossRef]

8. Shiraki N, Wakabayashi T, Sakaguchi H, Nishida K. Optical biometry-based intraocular lens calculation and refractive outcomes after phacovitrectomy for rhegmatogenous retinal detachment and epiretinal membrane. Sci Rep 2018;8:11319.

9. Abou-Shousha M, Helaly HA, Osman IM. The accuracy of axial length measurements in cases of macula-off retinal detachment. Can J Ophthalmol 2016;51:108-12. [CrossRef]

10. Kang TS, Park HJ, Jo YJ, Kim JY. Long-term reproducibility of axial length after combined phacovitrectomy in maculasparing rhegmatogenous retinal detachment. Sci Rep 2018;8:I5856.

II. Huang C, Zhang T, Liu J, Ji Q, Tan R. Changes in axial length, central cornea thickness, and anterior chamber depth after rhegmatogenous retinal detachment repair. BMC Ophthalmol 2016;16:12I. [CrossRef]

12. Kang EC, Lee $\mathrm{KH}$, Koh HJ Comparison of refractive error in phacovitrectomy for epiretinal membrane using ultrasound and partial coherence interferometry. Eur J Ophthalmol 
2016;26:356-60. [CrossRef]

13. Hosoda Y, Kuriyama S, Hattori H, Hayashi H, Matsumoto M. Outcome of primary vitrectomy in phakic patients aged younger than 50 years with rhegmatogenous retinal detachments. Jpn J Ophthalmol 2016;60:395-400. [CrossRef]

14. Soliman MK, Hardin JS, Jawed F, Uwaydat SH, Faramawi MF, Chu CJ, et al. A database study of visual outcomes and intraoperative complications of postvitrectomy cataract surgery. Ophthalmology 2018;125:1683-91. [CrossRef]

15. Manvikar SR, Allen D, Steel DHW. Optical biometry in combined phacovitrectomy. J Cataract Refract Surg 2009;35:64-9.

16. Ozal SA, Kupelı A, Ozal E, Gurlu V. Optical biometry-based axial length alterations after intravitreal dexamethasone implant. Arq Bras Oftalmol 2019;82:195-9. [CrossRef]

17. Prinz A, Neumayer T, Buehl W, Kiss B, Sacu S, Drexler W, et al. Influence of severity of nuclear cataract on optical biometry. J Cataract Refract Surg 2006;32:116I-5. [CrossRef]
18. Freeman G, Pesudovs K. The impact of cataract severity on measurement acquisition with IOL Master. Acta Ophthalmol Scand 2005;83:439-42. [CrossRef]

19. Shajari M, Cremonese C, Petermann K, Singh P, Müller M, Kohnen T. Comparison of axial length, corneal curvature, and anterior chamber depth measurements of 2 recently introduced devices to a known biometer. Am J Ophthalmol 2017;178:5864. [CrossRef]

20. Srivannaboon S, Chirapapaisan C, Chonpimai P, Loket S. Clinical comparison of a new swept-source optical coherence tomography-based optical biometer and a time-domain optical coherence tomography-based optical biometer. J Cataract Refract Surg 2015;41:2224-32. [CrossRef]

21. Zaldivar R, Shultz MC, Davidorf JM, Holladay JT. Intraocular lens power calculations in patients with extreme myopia. J Cataract Refract Surg 2000;26:668-74. [CrossRef] 